\title{
Intensive Care Unit Management of a Patient with Tracheal Rent Repair Following Laryngopharyngoesophagectomy
}

\author{
Jenna Arora ${ }^{1}$, Lalit Sehgal ${ }^{2}$, Himanshu Satpathy ${ }^{3}$
}

\begin{abstract}
Tracheal injuries are one of the potentially fatal complications following laryngopharyngeal and esophageal surgeries. The patient developed tracheal rent during laryngopharyngoesophagectomy. The injury was diagnosed intraoperative and repaired. However, it did not heal, and the patient developed tracheopleural fistula. Right thoracotomy and latissimus dorsi flap was done under general anesthesia. Postsurgery, the patient was shifted to intensive care unit (ICU), where he developed respiratory distress not improving, with increasing oxygen flows. To avoid damage to the repair, under bronchoscopic guidance bilateral selective mainstem bronchial intubations were done using cuffed $5.0 \mathrm{~mm}$ regular endotracheal tubes (ETTs), and ventilation was supported on pressure control ventilation mode. The ventilator support was weaned off to pressure support ventilation mode on postoperative day (POD) 1. On POD2, ETTs were removed under bronchoscopic guidance and were replaced by $7 \mathrm{~mm}$ ID long and adjustable flange tracheostomy tube with the tip just above the carina. The cuff was kept deflated, and oxygen with the high flow was provided through a tracheostomy. The high flow was weaned off after 5 days. Later, the patient was managed conservatively by regular chest physiotherapy, antibiotics, bronchoscopic pulmonary toileting, nebulizations, and appropriate antimicrobial therapy. Patient was discharged in stable condition from ICU and hospital.
\end{abstract}

Keywords: Bilateral selective mainstem bronchial intubation, High-flow oxygen delivery devices, Laryngopharyngoesophagectomy, Tracheal rent, Tracheopleural fistula.

Indian Journal of Critical Care Medicine (2020): 10.5005/jp-journals-10071-23332

\section{INTRODUCTION}

Tracheal injuries during surgeries are rare but may be one of the most lethal complications in the perioperative period. ${ }^{1}$ Early diagnosis and management can reduce the mortality. Most common presenting features of tracheal injuries are dyspnea, subcutaneous emphysema, hemoptysis, sternal tenderness, and pneumothorax. ${ }^{2}$ The management of such cases is always challenging. Furthermore, the options for airway management are limited. Here, we present successful intensive care unit (ICU) management of a patient following tracheal repair, who had developed tracheal tear during laryngopharyngoesophagectomy.

\section{Case Description}

The patient, a 56-year-old male, was a known case of carcinoma of post cricoid region (hypopharynx). He had received 70 cycles of chemotherapy and 35 cycles of radiotherapy. He underwent total laryngopharyngoesophagectomy with total thyroidectomy with central compartment clearance with a tracheostomy with gastric pull-up with pectoralis major myocutaneous flap with feeding jejunostomy under general anesthesia. Tracheal rent occurred during surgical dissection, which was recognized and repaired. The patient was shifted to ICU with armored endotracheal tube (ETT) placed through a tracheostomy. On postoperative day (POD) 1, bronchoscopic-guided long-flanged tracheostomy tube (TT) was placed, and cuff was kept deflated.

On POD5, rent measuring $1 \mathrm{~cm} \times 1 \mathrm{~cm}$ in posterior tracheal wall approximately $1.5-2 \mathrm{~cm}$ above carina was observed on check bronchoscopy. On POD6, the patient developed subcutaneous emphysema due to tracheopleural fistula (Fig. 1). He was posted for right thoracotomy with latissimus dorsi flap the next day under general anesthesia. During the procedure, the ventilation was managed with a left endobronchial tube via tracheostomy.
${ }^{1-3}$ Department of Anesthesiology, Rajiv Gandhi Cancer Institute and Research Centre, New Delhi, India

Corresponding Author: Lalit Sehgal, Department of Anesthesiology, Rajiv Gandhi Cancer Institute and Research Centre, New Delhi, India, Phone: +91 9910256127, e-mail: sehgal.lalit@gmail.com

How to cite this article: Arora J, Sehgal L, Satpathy H. Intensive Care Unit Management of a Patient with Tracheal Rent Repair Following Laryngopharyngoesophagectomy. Indian J Crit Care Med 2020;24(1):77-79.

Source of support: Nil

Conflict of interest: None

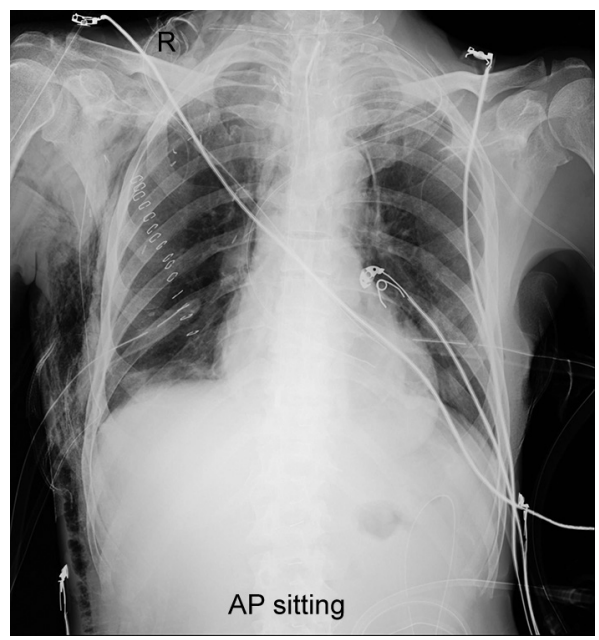

Fig. 1: X-ray chest anteroposterior view showing the presence of surgical emphysema following development of tracheopleural fistula 

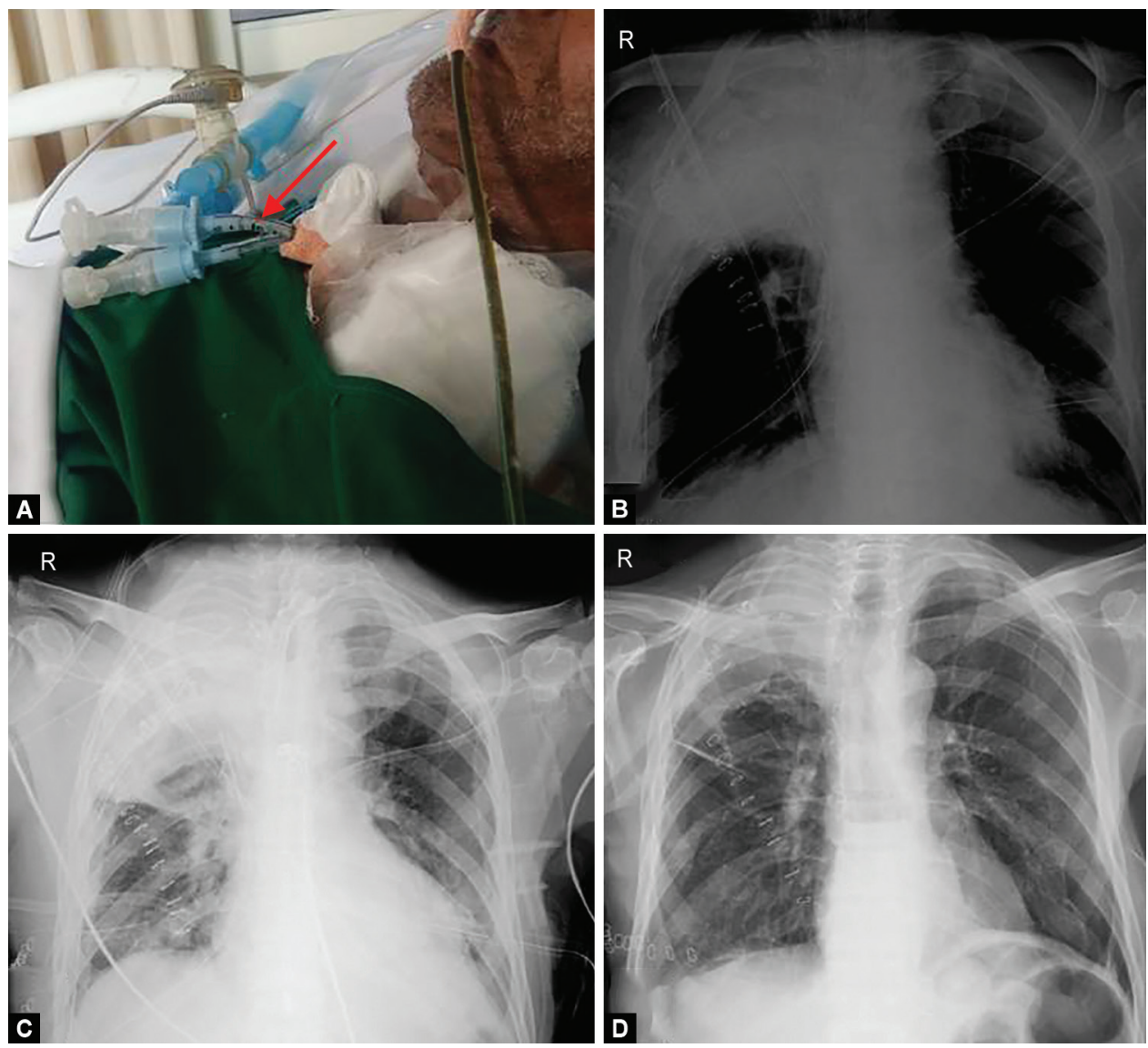

Figs 2A to D: (A) Conventional ETTs connected through a DLT connector to ventilator circuit; (B) chest X-ray POD1 depicting right upper lobe lung collapse along with ETT tip position in right mainstem bronchus. The left mainstem bronchus ETT is masked by cardiac silhouette; (C) chest X-ray on POD3 with TT in situ; (D) chest X-ray post decannulation

After surgery, neuromuscular blockade was reversed, spontaneous ventilation was resumed, and the endobronchial tube was removed, and oxygen via tracheal mask was provided. The patient was shifted to surgical ICU. He developed rapid shallow breathing, desaturation, and drowsiness. Increasing oxygen flow did not improve his condition. Under bronchoscopic guidance, $5.0 \mathrm{~mm} I \mathrm{D}$, cuffed ETT was placed in the right mainstem bronchus through a tracheostomy. The cuff of the tube was placed below the rent and was intended to keep the right upper lobe ventilated. Another cuffed ETT $5.0 \mathrm{~mm}$ ID was placed in left mainstem bronchus under bronchoscopic guidance. Tubes were fixed at $14 \mathrm{~cm}$ (left) and $13 \mathrm{~cm}$ (right) at the level of stoma and connected via a DLT connector to a ventilator (pressure control mode) (Figs $2 \mathrm{~A}$ to $\mathrm{D}$ ). Oxygen saturation improved thereafter.

Ventilator support was weaned off later to the spontaneous mode by the next day. Patient received intravenous morphine infusion. On POD1, right upper lobe collapse was noted on chest X-ray (Fig. 2B). Bronchoscopy was done to recheck tube position along with pulmonary toileting.

On POD2 of thoracotomy, the ETTs were removed under bronchoscopic guidance and were replaced by $7.0 \mathrm{~mm}$ ID long and adjustable flange TT. The tip of the tube was kept just above the carina, and cuff was kept deflated. Patient was maintained on high-flow oxygen (AIRVO2 ${ }^{\text {TM }}$ Fisher and Paykel) via tracheostomy, which was weaned off after 5 days. Later, patient was managed conservatively by chest physiotherapy, antibiotics, regular bronchoscopic pulmonary toileting, nebulizations, and appropriate antimicrobial therapy. Tracheal rent was seen healing, and granulation tissue was present on a repeat bronchoscopy on POD9 following thoracotomy. Patient was discharged in stable condition from ICU on POD23 and hospital on POD30 of thoracotomy.

\section{Discussion}

Tracheal tear poses a real challenge in most cases, as diagnosis may be delayed. ${ }^{3}$ In the present case, the damage was identified during the surgery itself and repaired. However, the patient developed a tracheopleural fistula on POD6. This could have happened due to poor healing because of the post-chemoradiotherapy status of the patient. ${ }^{4}$ During repair, left mainstem bronchus was selectively intubated to facilitate one-lung ventilation during the right thoracotomy. It may seem that the right lung was not expanded postsurgery, possibly leading to desaturation. Conservative management was not successful. The challenge was to ventilate the lungs without causing any pressure on the area of repair to allow healing. The air leak from the site of rupture can spread through the mediastinum and along the great vessels. It can even cause cardiac tamponade by spreading into the pericardium. ${ }^{5}$ Therefore, it is advisable to place the ETT/TT distal to the repair. The other alternatives for maintaining ventilation/oxygenation could be high-frequency oscillation ventilation, high-frequency jet ventilation, and extracorporeal membrane oxygenation. ${ }^{6,7}$ However, due to limited availability and experience of using, these modalities were not considered. Moreover, jet ventilation through a tracheostomy may still impair the healing. ${ }^{7}$ 
In the present case, the situation was complex due to the presence of rent in the distal trachea close to the carina. The options for managing ventilation are scarce. Selective bilateral endobronchial intubation through tracheostomy was done using conventional ETT. The cuff of the right endobronchial tube was positioned at carina partly in the trachea and right mainstem bronchus. It was done to ensure ventilation to the right upper lobe without compromising the repair. However, the chest X-ray done the next day revealed the right upper lobe collapse, which responded to bronchoscopic pulmonary toileting (Figs $2 \mathrm{~B}$ and $\mathrm{C}$ ). Wallet et al. have reported successful management of acute respiratory distress syndrome (ARDS) in the presence of tracheal injury by using selective bilateral mainstem bronchus intubation with small ETTs through a large tracheostomy. ${ }^{7}$ The authors utilized jet ventilation during the placement of the endobronchial tube before initiating protective lung ventilation. However, we did not have jet ventilation available.

Small ETTs have the potential for providing higher resistance to spontaneous breathing. ${ }^{8}$ Moreover, there are higher chances of blockage due to mucus plugs. The authors successfully utilized oxygen via high flow through a tracheostomy cannula to bridge once patient was on the spontaneous mode of ventilation through a long and adjustable flange TT. The tip of the TT was placed just above carina under bronchoscopic guidance, and cuff was kept deflated. Oxygen via high-flow nasal cannula has been found to be more successful over NIV in the management of early ARDS. ${ }^{9}$ In a crossover study, high-flow oxygen via tracheostomy was observed to improve oxygenation following prolonged mechanical ventilation. ${ }^{10}$ Mitaka et al. reported successful utilization of high-flow oxygen via tracheostomy for weaning after prolonged mechanical ventilation in two patients with restrictive pulmonary dysfunction. ${ }^{11}$

Sustained flow allowed the patient to breathe comfortably without creating any additional pressure to impair the healing. The preservation of spontaneous ventilation has been utilized in patients who are managed conservatively. ${ }^{12}$ The weaning methodology after initial stabilization was not mentioned in other reports.

To summarize, tracheal injury is a rare and potentially fatal complication of laryngopharyngoesophagectomy, which should be managed immediately with proactive maximal use of available resources.

\section{References}

1. Prokakis C, Koletsis EN, Dedeilias P, Fligou F, Filos K, Dougenis D. Airway trauma: a review on epidemiology, mechanisms of injury, diagnosis and treatment. J Cardiothorac Surg 2014;9:117. DOI: 10.1186/1749-8090-9-117.

2. Ye D, Shen Z, Zhang Y, Qiu S, Kang C. Clinical features and management of closed injury of the cervical trachea due to blunt trauma. Scand J Trauma Resusc Emerg Med 2013;21:60. DOI: 10.1186/1757-7241-21-60.

3. Hwang JJ, Kim YJ, Cho HM, Lee TY. Traumatic tracheobronchial injury: delayed diagnosis and treatment outcome. Korean J Thorac Cardiovasc Surg 2013;46(3):197-201. DOI: 10.5090/kjtcs.2013.46.3.197.

4. Machuzak MS, Santacruz JF, Jaber W, Gildea TR. Malignant trachealmediastinal-parenchymal-pleural fistula after chemoradiation plus bevacizumab: management with a Y-silicone stent inside a metallic covered stent. J Bronchology Interv Pulmonol 2015;22(1):85-89. DOI: 10.1097/LBR.0000000000000099.

5. Farooqui AM, Mbarushimana S, Faheem M. Unusual case of acute tracheal injury complicated by application of positive end expiratory pressure (PEEP). BMJ Case Rep 2014;2014:bcr-2014-206882. DOI: 10.1136/ bcr-2014-206882.

6. Hatipoglu Z, Turktan M, Avci A. The anesthesia of trachea and bronchus surgery. J Thorac Dis 2016;8(11):3442-3451. DOI: 10.21037/ jtd.2016.11.35.

7. Wallet $F$, Schoeffler M, Duperret S, Robert MO, Workineh S, Viale JP. Management of low tracheal rupture in patients requiring mechanical ventilation for acute respiratory distress syndrome. Anesthesiology 2008;108(1):159-162. DOI: 10.1097/01.anes.0000296104.46682.ca.

8. Farrow S, Farrow C, Soni N. Size matters: choosing the right tracheal tube. Anaesthesia 2012;67(8):815-819. DOI: 10.1111/j.13652044.2012.07250.x.

9. Frat JP, Coudroy R, Marjanovic N, Thille AW. High-flow nasal oxygen therapy and noninvasive ventilation in the management of acute hypoxemic respiratory failure. Ann Transl Med 2017;5(14):297. DOI: 10.21037/atm.2017.06.52.

10. Corley A, Edwards M, Spooner AJ, Dunster KR, Anstey C, Fraser JF. High-flow oxygen via tracheostomy improves oxygenation in patients weaning from mechanical ventilation: a randomised crossover study. Intensive Care Med 2017;43(3):465-467. DOI: 10.1007/ s00134-016-4634-7.

11. Mitaka C, Odoh M, Satoh D, Hashiguchi T, Inada E. High-flow oxygen via tracheostomy facilitates weaning from prolonged mechanical ventilation in patients with restrictive pulmonary dysfunction: two case reports. J Med Case Rep 2018;12(1):292. DOI: 10.1186/s13256018-1832-7.

12. Conti $M$, Pougeoise $M$, Wurtz A, Porte $H$, Fourrier F, Ramon $P$, et al. Management of postintubation tracheobronchial ruptures. Chest 2006;130(2):412-418. DOI: 10.1378/chest.130.2.412. 